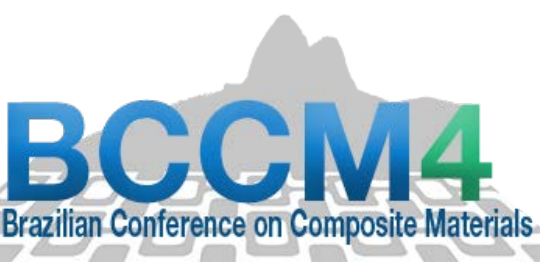

\title{
CRITICAL REVIEW OF DISPLACEMENT-BASED LAMINATE THEORIES AND MODELING TECHNIQUES
}

\author{
$\underline{\text { André S. de Lima }}^{(1)}$, Alfredo R. de Faria ${ }^{(1)}$ and José J. R. Faria ${ }^{(1)}$
}

(1) Department of Aeronautical and Mechanical Engineering, Instituto Tecnológico de Aeronáutica, Brazil

https://doi.org/doi/10.21452/bccm4.2018.01.02

\begin{abstract}
Composite materials present many particularities and challenges when it comes to structural modelling. These challenges come from the anisotropic and nonhomogeneous nature of these materials combined with the continuity requirements of displacement and transverse stresses through the thickness, commonly named $\mathrm{C}_{z}^{0}$ requirements. The different approaches developed to assess the structural behaviour of composites can be divided into two major branches: displacement-based theories and mixed-formulation theories. Among the displacement-based theories, the most common approaches are: Equivalent Single Layer (ESLT), that includes the Classical Laminate Theory (CLT); Layerwise Theories (LT), that includes the Zig-Zag Theories (ZZT); Quasi-Layerwise Theories (QLT); and Global-Local Superposition Theories (GLST). The present paper discusses how these different displacement-based models handle the particularities of structural composites, highlighting the main advantages and disadvantages of each formulation, as well as their applicability to common structural problems.
\end{abstract}

\section{INTRODUCTION}

The nonhomogeneous nature of composite materials pose a series of particularities regarding their structural behaviour, especially in the transverse (through-the-thickness) direction. This characteristic, combined with the principles of macro-mechanics, result in the inherited $\mathrm{C}^{0}$ continuity requirements of transverse displacements and stresses. These are commonly named $\mathrm{C}_{z}^{0}$ continuity requirements [1], where the subscript $z$ denotes the transverse direction. Moreover, because of the nonhomogeneous characteristic on that direction, in-plane displacements and transverse shear stresses usually present kinks (discontinuities on the first derivative) in the interfaces between laminas. Therefore, a robust composite theory must account for these effects.

Investigating the history of two-dimensional composite modelling techniques, one can conclude that there has been a gradual evolution of laminate theories, in order to account for the aforementioned particularities, which cannot be fully represented by classical plate and beam theories. The current study presents a critical review of the 
displacement-based theories in contrast to those inherited requirements of composite structures. It is noteworthy to mention that the present work is of introductory nature. A number of authors have conducted extensive and comprehensive reviews on this topic, namely Liu and Li [2], Carrera [3,4] and more recently Sayyad and Ghugal [5,6].

\section{DISPLACEMENT-BASED FORMULATIONS}

The displacement-based theories receive this name because the primary variables are displacement-related and compose the displacement field assumed by the theory. Departing from their displacement field and assuming strain-displacement and constitutive relationships, the stresses and strains are determined.

The displacement field of most displacement-based formulations are a combination of one or more fields illustrated on Figure 1.

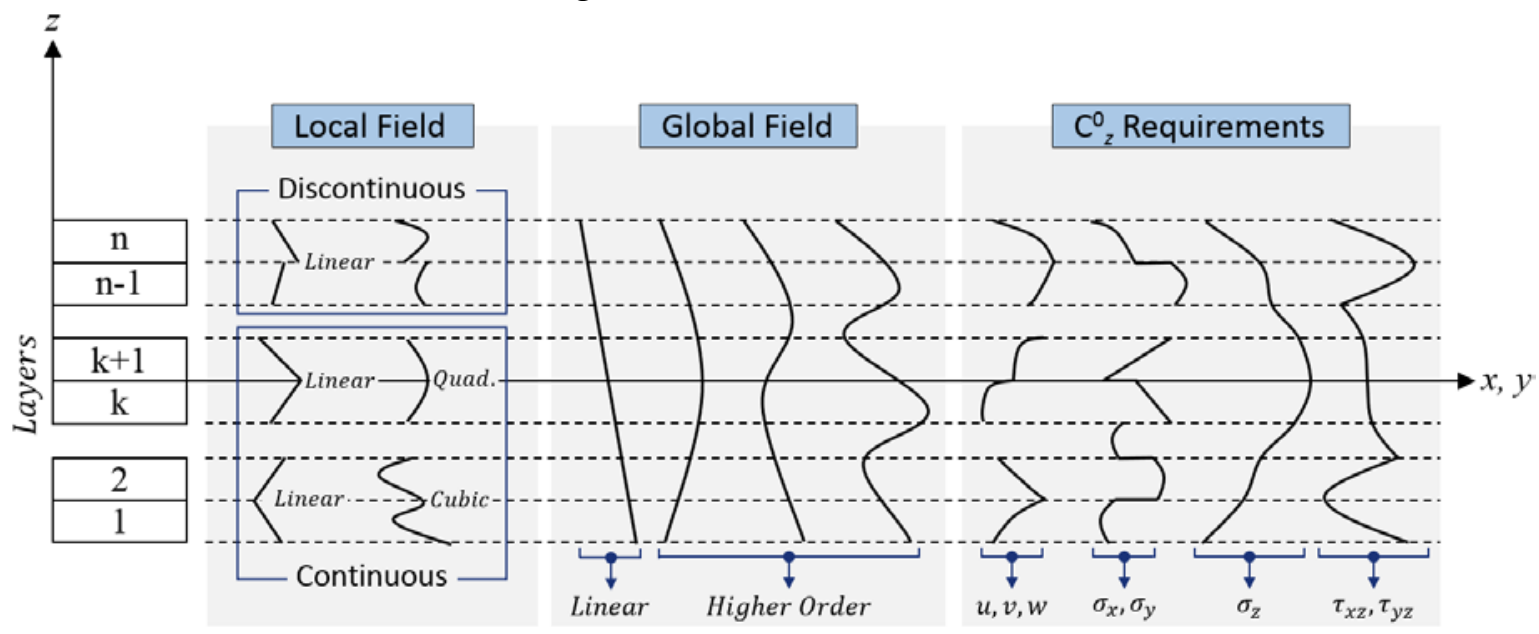

Figure 1: Comparison of assumed displacement fields (local and global) and $\mathrm{C}^{0}{ }_{\mathrm{z}}$ requirements.

In Figure 1, the displacement fields are represented by polynomial functions only; however, other types of functions, such as exponential, trigonometric and hyperbolic, were also used to develop displacement-based theories [5], [6].

The next sections present, in a very succinct and general form, an overview of the most common two-dimensional displacement-based formulations applied to composite laminates.

\subsection{Equivalent Single Layer Theories}

The first attempts to model composite structures used traditional plate theories, where the displacement field was assumed smoothly continuous along the thickness, implicating in a transverse global behaviour independent of the number of layers, thus treating the laminate as a single layer. Therefore, these theories are commonly classified as Equivalent Single Layer Theories (ESLT).

In this universe, the Classical Laminate Theory (CLT) is based on the straightforward application of Kirchhoff's plate theory [7] to laminates, whose displacement field is presented in Table 1. One outstanding deficiency of the CLT is the assumption of zero transverse shear. In order to overcome this serious limitation constant shear theories were developed applying Reissner [8] and Mindlin [9] formulations to laminated plates. Because the polynomials used in these theories are linear in the $z$-coordinate (through the thickness) and the predicted shear is constant, they are commonly referred to as first-order shear deformation theories. 
Subsequently, several researches proposed theories where the displacement fields were described with higher-order polynomials, giving rise to Higher-Order Shear Deformation Theories (HSDT). A generic expression of the HSDT is also presented in Table 1.

Table 1: CLT and HSDT displacement fields

\begin{tabular}{cc}
\hline CLT & HSDT \\
\hline$u(x, y, z)=u_{0}(x, y)-\frac{d w}{d x} z(x, y, z)=\sum_{i=0}^{m} u_{i}(x, y) z^{i}$ \\
$v(x, y, z)=v_{0}(x, y)-\frac{d w}{d y} z$ & $v(x, y, z)=\sum_{i=0}^{m} v_{i}(x, y) z^{i}$ \\
$w(x, y, z)=w_{0}(x, y)$ & $w(x, y, z)=\sum_{i=0}^{m} w_{i}(x, y) z^{i}$ \\
\hline
\end{tabular}

Sayyad and Ghugal [4,5] recently conducted extensive reviews on beam and plate theories for composite plates with more than 800 references. From this extensive compilation, it is observed that not only polynomial functions were used to express displacement fields, but also exponential, hyperbolic and trigonometric functions, as previously mentioned. One can also observe that many theories consider transverse shear strains, but neglect the transverse normal strain, keeping the traditional homogeneous plate assumption that the transverse displacement $w$ is constant through the thickness. This implicates in the inability to determine the transverse normal stress $\sigma_{z}$ directly from the constitutive relationships, requiring its recovery via integration of the equilibrium equation (Eq. 1). In fact, only a small number of theories account for transverse normal deformation, which in many applications is important. Two examples of such applications are delamination prediction and thermo-mechanical analysis.

$\sigma_{z, z}+\tau_{x z, x}+\tau_{y z y}=0$

The major benefit of this class of theories is the low computational burden. On the other hand, the major drawbacks are is the inability of describing zig-zag distribution (kinks) of in-plane displacements, as illustrated in Figure 1. This implies in continuous strains through-the-thickness and in double-valued transverse stresses on the lamina interfaces, regardless of the order of ESLT.

\subsection{Layerwise Theories}

In a broad sense, Layerwise Theories (LT) encompass all theories that assume a local displacement field expansion within each material layer. By doing so, these theories allow for the computation of zig-zag shaped displacements and continuous transverse stresses through-the-thickness, better predicting the overall behaviour of laminated composites. However, this is not achieved without penalties, as computational efficiency is sacrificed in exchange for numerical accuracy. Carrera [4] conducted a broad historical review of LTs (referred to by the author as Zig-Zag Theories), which highlights the importance of the early studies developed by Lekhnitskii [10], Ambartsumian [11] and Reissner [12]. According to Carrera, most of the subsequent developments of laminate LTs are somewhat refinements of these original studies, despite the lack of recognition in the open literature, particularly for Lekhnitskii's work. 
Lee and Liu [13] proposed a full LT of third-order assigning a translational component and its first derivatives as variables for each layer surface and using Hermite cubics as interpolation functions in order to model the displacement field of a composite layer. According to Liu and Li [2], the results from Lee and Liu's full LT [13] are believed to be the most accurate among all the laminate theories available in the literature.

Following the approach of Liu and Li [2], a generalized form of the displacement field of the third-order LT can be written as follows:

$$
\begin{aligned}
& u^{k}(x, y, z)=u_{0}^{k}(x, y)+u_{1}^{k}(x, y) z+u_{2}^{k}(x, y) z^{2}+u_{3}^{k}(x, y) z^{3} \\
& v^{k}(x, y, z)=v_{0}^{k}(x, y)+v_{1}^{k}(x, y) z+v_{3}^{k}(x, y) z^{2}+v_{3}^{k}(x, y) z^{3} \\
& w^{k}(x, y, z)=w_{0}^{k}(x, y)+w_{1}^{k}(x, y) z+w_{3}^{k}(x, y) z^{2}+w_{3}^{k}(x, y) z^{3}
\end{aligned}
$$

Notice that the displacement fields presented in Eq. 2 possess four variables in each component, totalizing $12 n$ variables for a laminate with $n$ layers. The total number of variables can be reduced by imposing displacement $(u, v, w)$ and transverse stresses $\left(\tau_{x z}\right.$, $\left.\tau_{y z}, \sigma_{z}\right)$ continuity at every lamina interface, yielding $6(n+1)$ variables. This not only reduces the total number of variables, but significantly improves overall accuracy [13]. Additionally, by imposing transverse stress boundary conditions on the upper and lower surfaces of the laminate, the number of variables can be further reduced to $6 n$. It is possible to notice the resemblance of Eq. 2 with the HSDT formulation presented in Table 1. In fact, the HSDT can be seen as a simplified version of the LT.

\subsubsection{Zig-Zag Theories}

As one may anticipate, the term Zig-Zag Theory (ZZT) is granted due to the zig-zag distribution of in-plane displacements through-the-thickness attained by these formulations. They are a particular type of LT, in the sense that they make use of global and layer-dependent (local) displacement components. A number of early ZZTs were proposed assuming in-plane displacement continuity only [10-12]. These theories improved in-plane stress prediction, but transverse shear stresses were still constant through-the-thickness, due to the low order of the assumed displacement fields. Later developments also failed to predict transverse shear stresses by employing higher-order displacement terms, and it was not until Cho and Parmerter [17] that transverse stress continuity conditions were accounted for and correct transverse shear stresses were predicted directly from constitutive relations.

Liu and $\mathrm{Li}$ [2] unified the notations of the ZZTs by expressing the displacement fields as:

$$
\begin{aligned}
& u^{k}(x, y, z)=u_{0}^{k}(x, y)+u_{1}^{k}(x, y) z+u_{2}(x, y) z^{2}+u_{3}(x, y) z^{3} \\
& v^{k}(x, y, z)=v_{0}^{k}(x, y)+v_{1}^{k}(x, y) z+v_{2}(x, y) z^{2}+v_{3}(x, y) z^{3} \\
& w^{k}(x, y, z)=w_{0}(x, y)
\end{aligned}
$$

Other ZZTs were developed by changing the order of the layer-dependent terms (i.e. zeroth and second order, first and second order, etc.), but in all of them, there are only two layer-dependent terms. This is because using two layer-dependent terms, the number of layer-dependent variables $(4 n)$ is equal to the number of the displacement $(u$, $v$ ) and transverse stresses $\left(\tau_{x z}, \tau_{y z}\right)$ continuity equations $(4 n-4)$, plus the boundary conditions for the transverse stresses on the upper and lower surfaces (4). As a result, all layer dependent variables can be eliminated. 
Because transverse deformation is neglected, the continuity equations related to $w$ and $\sigma_{z}$ are not available, as in the full LTs. These theories are also referred to as QuasiLayerwise Theories (QLT) [2].

One drawback of this class of theories is that the predicted transverse shear stress is smooth through-the-thickness, failing to capture distinct kinks in lamina interfaces (See Figure 1) even when employing higher-order local displacement components. Moreover, the $w$ displacement continues to be assumed constant through-the-thickness, meaning that the transverse normal strain is neglected, and the transverse normal stress can only be recovered from the integration of the equilibrium equations. Also, there is a dependency of the definition of the local coordinate system for the layers [2], reducing the robustness of such theories.

\subsubsection{Superposition Theories}

In the light of the improvements achieved by these so-called ZZTs, it became evident that an attractive strategy to develop an accurate and computationally efficient theory for laminated plates would be to combine the layer-independence of HSDTs with the capability of predicting layerwise behaviour, while also preserving the capability to determine transverse stresses directly from the constitutive equations, without the need of postprocessing. Liu and Li [18] proposed such an approach by introducing global and local displacement components based on a generalized coordinate system, named Superposition Theory (ST).

The displacement field of the kth layer of a laminated composite plate can be expressed in terms of global and local (layer-dependent) components as:

$$
\begin{aligned}
& u^{k}=u_{G}+u_{L}^{k} \\
& v^{k}=v_{G}+v_{L}^{k} \\
& w^{k}=w_{G}
\end{aligned}
$$

where $k$ is the layer order and the subscripts $L$ and $G$ represent local and global components, respectively. Departing from Eq. 3 (also known as 0-1 QLT) and Eq. 4, the 0-1 ST can be written as:

$$
\begin{aligned}
& u^{k}(x, y, z)=u_{0}(x, y)+u_{1}(x, y) z+u_{2}(x, y) z^{2}+u_{3}(x, y) z^{3}+u_{0}^{k}+u_{1}^{k} \xi_{k} \\
& v^{k}(x, y, z)=v_{0}(x, y)+v_{1}(x, y) z+v_{2}(x, y) z^{2}+v_{3}(x, y) z^{3}+v_{0}^{k}+v_{1}^{k} \xi_{k} \\
& w^{k}(x, y, z)=w_{0}(x, y)
\end{aligned}
$$

Notice that for global displacement terms the $z$-coordinate is used, while for local description, a linear coordinate $\xi_{k}$ for the $k$ th layer is used. This is the fundamental difference between ZZTs, or QLTs, and STs, which renders the biggest advantages of STs: independence of the number of layers and of the definition of the local coordinate system.

As for the QLTs, STs can be classified by the order of the local terms (i.e. 0-1, 0-2, $0-3,1-2,1-3,2-3$ ). The 1-3 ST (local terms are of first and third-order) is considered the best among the other six investigated theories of this family, due to its excellent predictions of $\tau_{x z}$ and $\tau_{y z}$ [2]. This indicates that the first and third-order terms play an important role on the overall accuracy, both for displacement and stresses. According to Liu and $\mathrm{Li} \mathrm{[2]} \mathrm{a} \mathrm{possible} \mathrm{interpretation} \mathrm{is} \mathrm{that} \mathrm{the} \mathrm{first-order} \mathrm{layer} \mathrm{dependent} \mathrm{term} \mathrm{is}$ considered the most fundamental one (associated with rotational angle), while the thirdorder term is particularly important in order to predict the layerwise parabolic distribution of the shear stress. Nevertheless, with the exception of the zeroth-order 
terms, which can be accurately represented globally, all local terms have a distinct contribution to laminate performance.

\subsubsection{Double-Superposition Theories}

In view of a more accurate representation of the laminate behaviour, accounting for as many local terms as possible, Liu and $\mathrm{Li}$ [2] propose the Double Superposition Theory (DBST), which consists of applying the ST technique twice to three local terms. This is necessary since only two continuity conditions can be satisfied in composite layer assembly, limiting the number of variables associated to local behaviour to two, as noted in Section 2.2.1. The displacement field then assumes the following general form:

$$
\begin{aligned}
u^{k} & =u_{\mathrm{G}}+\bar{u}_{\mathrm{L}}^{k}+\tilde{u}_{\mathrm{L}}^{k} \\
v^{k} & =v_{\mathrm{G}}+\bar{v}_{\mathrm{L}}^{k}+\tilde{v}_{\mathrm{L}}^{k} \\
w^{k} & =w_{\mathrm{G}}
\end{aligned}
$$

The local terms can have different arrangements of first, second and third-order, giving rise to different theories, for example the 1,2-3 DBST:

$$
\begin{aligned}
& \bar{u}_{\mathrm{L}}^{k}\left(x, y, \xi_{k}\right)=u_{1}^{k}+u_{2}^{k} \xi_{k}{ }^{2} ; \tilde{u}_{\mathrm{L}}^{k}\left(x, y, \xi_{k}\right)=u_{3}^{k} \xi_{k}{ }^{3} \\
& \bar{v}_{\mathrm{L}}^{k}\left(x, y, \xi_{k}\right)=v_{1}^{k}+v_{2}^{k} \xi_{k}{ }^{2} ; \tilde{v}_{\mathrm{L}}^{k}\left(x, y, \xi_{k}\right)=v_{3}^{k} \xi_{k}{ }^{3}
\end{aligned}
$$

According to Liu and $\mathrm{Li}$ [2], the results of the different arrangements of DBST are equivalent. Although an excellent prediction of displacements and transverse stresses, there is still the need of post-processing to recover the transverse normal stress for ST and DBST. The reason for this is that the variation of $w$ through-the-thickness is still neglected or assumed as a global distribution. This is a common aspect to most of the presented formulations, with the exception of full layerwise theories, which assume the general form of Eq. 2.

In an effort to overcome this drawback, Lima and Faria [19] proposed a 0-1 ST formulation for a two-dimensional beam finite element considering a local $w$ displacement distribution. There was a satisfactory response of the displacements, inplane stress and transverse shear stress, but the predicted transverse normal strain and stresses obtained directly from constitutive relations did not possess the same level of accuracy, and post-processing was required to correct the results. Despite the deficient accuracy of the later quantities, the element meets all $\mathrm{C}^{0}{ }_{z}$ requirements, with the exception of the kinks in transverse shear stress, handling non-homogeneous boundary conditions on the laminate surfaces and obtaining all stresses directly from the constitutive relations. Improved results are expected by increasing the order of the local terms, such as 1-3 ST, according to the observations of Liu and Li [2].

\section{SUMMARY AND CONCLUSIONS}

The previous sections gave an overview of the main displacement-based theories, presenting their displacement fields and capabilities to satisfy the so-called $\mathrm{C}_{z}^{0}$ continuity requirements, illustrated in Figure 1. In order to provide a clear comparison between the theories in a summarized manner, they were organized in a table form, and their main characteristics highlighted: 
Table 2: Comparison of the different displacement theories

\begin{tabular}{|c|c|c|c|c|c|}
\hline & CLT & HSDT & $\mathrm{ZZT}$ & GLT & $\mathrm{LT}$ \\
\hline $\begin{array}{c}\text { Displacement } \\
\text { Continuity }\end{array}$ & Yes & Yes & Yes & Yes & Yes \\
\hline Zig-zag shape & & & & & \\
\hline $\begin{array}{l}\text { of in-plane } \\
\text { disp. }\end{array}$ & No & No & Yes & Yes & Yes \\
\hline $\begin{array}{l}\text { Transverse } \\
\text { stresses } \\
\text { continuity }\end{array}$ & No & No & Yes & Yes & Yes \\
\hline $\begin{array}{c}\tau_{\mathrm{xz}} ; \tau_{\mathrm{yz}} \\
\text { accuracy }\end{array}$ & $\begin{array}{c}\tau_{\mathrm{xz}} ; \tau_{\mathrm{yz}} \\
\text { neglected }\end{array}$ & Low & $\begin{array}{l}\text { Good above 5-th } \\
\text { order. Dependent } \\
\text { coordinate system } \\
\text { definition. Smooth. }\end{array}$ & $\begin{array}{l}\text { Good when first } \\
\text { and third-order } \\
\text { local terms are } \\
\text { considered. } \\
\text { Kinks are captured. }\end{array}$ & Excellent \\
\hline $\begin{array}{l}\sigma_{\mathrm{z}} \text { accuracy } \\
\text { Dependency }\end{array}$ & Neglects & Postprocessed & Postprocessed & Postprocessed & Excellent \\
\hline $\begin{array}{c}\text { on the number } \\
\text { of layers }\end{array}$ & No & No & No & No & Yes \\
\hline
\end{tabular}

It becomes clear that laminated composite structures require a higher level of discretization when compared to homogeneous structures, since layerwise behaviour must be well represented. Therefore, there is a continuous effort in the academia to develop accurate and computational efficient theories capable of describing the behaviour of laminated composite structures. Among the discussed theories, the ones that present best compromise between numerical efficiency and accuracy are the ones based on the global-local superposition approach; however, there are still improvements to be done in the theories currently available in the open literature.

\section{ACKNOWLEDGEMENTS}

The authors would like to acknowledge the Brazilian funding agencies CAPES and CNPQ for the grants CAPES 1642839, CNPq 306193/2017-5 and 163101/2017-5 that supported this research.

\section{REFERENCES}

[1] E. Carrera, "A class of two-dimensional theories for anisotropic multilayered plates analysis," Atti della Accad. delle Sci. di Torino. Cl. di Sci. Fis. Mat. e Nat., vol. 19, pp. 49-87, 1995.

[2] D. Liu and X. Li, "An Overall View of Laminate Theories Based on Displacement Hypothesis,” J. Compos. Mater., vol. 30, no. 14, pp. 1539-1561, 1996.

[3] E. Carrera, Theories and finite elements for multilayered, anisotropic, composite plates and shells, vol. 9, no. 2. 2002.

[4] E. Carrera, "Historical review of Zig-Zag theories for multilayered plates and shells," Appl. Mech. Rev., vol. 56, no. 3, p. 287, 2003.

[5] A. S. Sayyad and Y. M. Ghugal, "On the free vibration analysis of laminated composite and sandwich plates: A review of recent literature with some numerical results," 
Compos. Struct., vol. 129, pp. 177-201, 2015.

[6] A. S. Sayyad and Y. M. Ghugal, "Bending, buckling and free vibration of laminated composite and sandwich beams: A critical review of literature,” Compos. Struct., vol. 171, pp. 486-504, 2017.

[7] G. Kirchhoff, "Ueber das Gleichgewicht und die Bewegung eines unendlich dünnen elastischen Stabes,” J. für die reine und Angew. Math., no. 56, pp. 285-313, 1859.

[8] E. Reissner, "On transverse bending of plates, including the effect of transverse shear deformation,” Int. J. Solids Struct., vol. 11, no. 5, pp. 569-573, 1975.

[9] R. D. Mindlin, "Influence of rotatory inertia and shear in flexural motions of isotropic elastic plates,” ASME J. Appl. Mech., vol. 18, pp. 1031-1036, 1951.

[10] S. G. Lekhnitskii, "Strength calculation of composite beams," Vestn. inzhen i tekhnikov, vol. 9, 1935.

[11] S. A. Ambartsumian, "On the theory of bending of anisotropic plates and shallow shells,” J. Appl. Math. Mech., vol. 24, no. 2, pp. 500-514, 1960.

[12] E. Reissner, "On a certain mixed variational theorem and a proposed application," Int. J. Numer. Methods Eng., vol. 20, no. 7, pp. 1366-1368, 1984.

[13] C. Y. Lee and D. Liu, "An interlaminar stress continuity theory for laminated composite analysis,” Comput. Struct., vol. 42, no. 1, pp. 69-78, 1992.

[14] M. di Sciuva, "Bending, vibration and buckling of simply supported thick multilayered orthotropic plates: An evaluation of a new displacement model,” J. Sound Vib., vol. 105, no. 3, pp. 425-442, 1986.

[15] H. Murakami, "Laminated Composite Plate Theory With Improved In-Plane Responses,” J. Appl. Mech., vol. 53, no. 3, pp. 661-666, Sep. 1986.

[16] E. J. Barbero, J. N. Reddy, and J. Teply, "An accurate determination of stresses in thick laminates using a generalized plate theory,” Int. J. Numer. Methods Eng., vol. 29, no. 1, pp. 1-14, 1990.

[17] M. Cho and R. R. Parmerter, "An efficient higher order plate theory for laminated composites,” Compos. Struct., vol. 20, no. 2, pp. 113-123, 1992.

[18] X. Li and D. Liu, "A laminate theory based on global-local superposition," Commun. Numer. Methods Eng., vol. 11, no. 8, pp. 633-641, 1995.

[19] A. Lima and A. Faria, "A composite beam element with through the thickness capabilities based on global-local superposition,” no. Ic, pp. 1-26. 